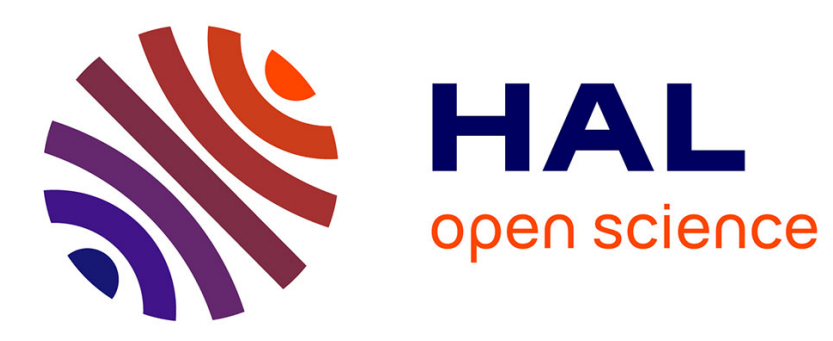

\title{
Games on lattices, multichoice games and the Shapley value: a new approach
}

\author{
Michel Grabisch, Fabien Lange
}

\section{To cite this version:}

Michel Grabisch, Fabien Lange. Games on lattices, multichoice games and the Shapley value: a new approach. Mathematical Methods of Operations Research, 2007, 65 (1), pp.153-167. 10.1007/s00186006-0109-x . halshs-00178916

\section{HAL Id: halshs-00178916 https://shs.hal.science/halshs-00178916}

Submitted on 12 Oct 2007

HAL is a multi-disciplinary open access archive for the deposit and dissemination of scientific research documents, whether they are published or not. The documents may come from teaching and research institutions in France or abroad, or from public or private research centers.
L'archive ouverte pluridisciplinaire HAL, est destinée au dépôt et à la diffusion de documents scientifiques de niveau recherche, publiés ou non, émanant des établissements d'enseignement et de recherche français ou étrangers, des laboratoires publics ou privés. 


\title{
Games on lattices, multichoice games and the Shapley value: a new approach
}

\author{
Michel GRABISCH*and Fabien LANGE \\ Université Paris I - Panthéon-Sorbonne \\ CERMSEM \\ 106-112, Bd de l'Hôpital, 75013 Paris, France \\ email Michel.Grabisch@lip6.fr, fabien.lange@univ-paris1.fr
}

\footnotetext{
${ }^{*}$ Corresponding author. Mailing address: LIP6, 8 rue du Capitaine Scott, 75015 Paris, France. Tel (+33) 1-44-27-88-65, Fax (+33) 1-44-27-70-00
} 


\begin{abstract}
Multichoice games, as well as many other recent attempts to generalize the notion of classical cooperative game, can be casted into the framework of lattices. We propose a general definition for games on lattices, together with an interpretation. Several definitions of the Shapley value of a multichoice games have already been given, among them the original one due to Hsiao and Raghavan, and the one given by Faigle and Kern. We propose a new approach together with its axiomatization, more in the spirit of the original axiomatization of Shapley, and avoiding a high computational complexity.
\end{abstract}

\title{
1 Introduction
}

The field of cooperative game theory has been enriched these recent years by many new kinds of game, trying to model in a more accurate way the behaviour of players in a real situation. In the classical view of cooperative games, to each coalition of players taking part into the game, an asset or a power (voting games) is associated, and participation is assumed to be of a binary nature, i.e., either a player participates or he does not. From this point, many variations have been introduced, let us cite games with precedence constraints among players (Faigle and Kern [6]) where not all coalitions are valid, ternary voting games (Felsenthal and Machover [7]) where abstention is permitted, bi-cooperative games (Bilbao [2]) where each player can choose to play either in favor, against, or not to play, multichoice games (Hsiao and Raghavan [14]) where each player has a set of $m$ possible ordered actions, fuzzy games (Butnariu and Klement [4], Tijs et al. [18]) which can be seen as a continuous generalization of multichoice games, global games (Gilboa and Lehrer [8]) where coalitions are replaced by partitions of the set of players, etc.

All the above examples of games can be casted into the general framework of games defined on a lattice, i.e., functions $v:(L, \leq) \longrightarrow \mathbb{R}$, where $(L, \leq)$ is a lattice, and such that $v(\perp)=0, \perp$ denoting the bottom element of $L$. We mention at this point that one can define games on other structures of discrete mathematics, such as matroids and convex geometries; this has been extensively studied by Bilbao [1].

A central question in game theory is to define a value or solution concept for a game, i.e. how to individually reward players supposing that all players have joined the grand coalition. A famous example for classical games is the Shapley value, based on rational axioms for sharing the total worth of the game $v(N)$. A different approach is to consider the core of the game, i.e., the set of imputations such that no subcoalition can do better by itself.

The aim of this paper is first to provide a general approach to games on lattices, giving an interpretation in terms of elementary actions, and second to provide a definition for the Shapley value together with an axiomatization. As it will be discussed, other previous definitions of the Shapley value have been given. We will focus on the works of Faigle and Kern [6], and Hsiao and Raghavan [14]. Previous works of the authors around this topic can be found in $[12,11,10]$. 


\section{Mathematical background}

We begin by recalling necessary material on lattices (a good introduction on lattices can be found in [5]), in a finite setting. A lattice is a set $L$ endowed with a partial order $\leq$ such that for any $x, y \in L$ their least upper bound $x \vee y$ and greatest lower bound $x \wedge y$ always exist. For finite lattices, the greatest element of $L$ (denoted $T$ ) and least element $\perp$ always exist. $x$ covers $y$ (denoted $x \succ y$ ) if $x>y$ and there is no $z$ such that $x>z>y$. The lattice is distributive if $\vee, \wedge$ obey distributivity. An element $j \in L$, $j \neq \perp$, is join-irreducible if it cannot be expressed as a supremum of other elements. Equivalently $j$ is join-irreducible if it covers only one element. Join-irreducible elements covering $\perp$ are called atoms, and the lattice is atomistic if all join-irreducible elements are atoms. The set of all join-irreducible elements of $L$ is denoted $\mathcal{J}(L)$.

An important property is that in a distributive lattice, any element $x$ can be written as an irredundant supremum of join-irreducible elements in a unique way (this is called the minimal decomposition of $x$ ). We denote by $\eta^{*}(x)$ the set of join-irreducible elements in the minimal decomposition of $x$, and we denote by $\eta(x)$ the normal decomposition of $x$, defined as the set of join-irreducible elements smaller or equal to $x$, i.e., $\eta(x):=\{j \in$ $\mathcal{J}(L) \mid j \leq x\}$. Hence $\eta^{*}(x) \subseteq \eta(x)$, and

$$
x=\bigvee_{j \in \eta^{*}(x)} j=\bigvee_{j \in \eta(x)} j
$$

Let us rephrase differently the above result. We say that $Q \subseteq L$ is a downset of $L$ if $x \in Q$ and $y \leq x$ imply $y \in Q$. For any subset $P$ of $L$, we denote by $\mathcal{O}(P)$ the set of all downsets of $P$. Then the mapping $\eta$ is an isomorphism of $L$ onto $\mathcal{O}(\mathcal{J}(L)$ ) (Birkhoff's theorem).

In a finite setting, Boolean lattices are of the type $2^{N}$ for some set $N$, i.e. they are isomorphic to the lattice of subsets of some set, ordered by inclusion. Boolean lattices are atomistic, and atoms corresponds to singletons. A linear lattice is such that $\leq$ is a total order. All elements are join-irreducible, except $\perp$.

Given lattices $\left(L_{1}, \leq_{1}\right), \ldots,\left(L_{n}, \leq_{n}\right)$, the product lattice $L=L_{1} \times \cdots \times L_{n}$ is endowed with the product order $\leq$ of $\leq_{1}, \ldots, \leq_{n}$ in the usual sense. Elements of $x$ can be written in their vector form $\left(x_{1}, \ldots, x_{n}\right)$. We use the notation $\left(x_{A}, y_{-A}\right)$ to indicate a vector $z$ such that $z_{i}=x_{i}$ if $i \in A$, and $z_{i}=y_{i}$ otherwise. Similarly $L_{-i}$ denotes $\prod_{j \neq i} L_{j}$. All joinirreducible elements of $L$ are of the form $\left(\perp_{1}, \ldots, \perp_{i-1}, j_{i}, \perp_{i+1}, \ldots, \perp_{n}\right)$, for some $i$ and some join-irreducible element $j_{i}$ of $L_{i}$. A vertex of $L$ is any element whose components are either top or bottom. We denote $\Gamma(L)$ the set of vertices of $L$. Note that $\Gamma(L)=L$ iff $L$ is Boolean, since in this case, denoting the trivial lattice $\{\perp, \top\}$ by 2 , we have $L=\underbrace{2 \times \cdots \times 2}_{n \text { times }}=2^{n}$.

\section{Games on lattices}

We denote by $N:=\{1, \ldots, n\}$ the set of players.

Definition 1 We consider finite distributive lattices $\left(L_{1}, \leq_{1}\right), \ldots,\left(L_{n}, \leq_{n}\right)$ and their product $L:=L_{1} \times \cdots \times L_{n}$ endowed with the product order $\leq$. A game on $L$ is any function 
$v: L \longrightarrow \mathbb{R}$ such that $v(\perp)=0$. The set of such games is denoted $\mathcal{G}(L)$. A game is monotone if $x \leq x^{\prime}$ implies $v(x) \leq v\left(x^{\prime}\right)$.

Lattice $\left(L_{i}, \leq_{i}\right)$ represents the (partially) ordered set of actions, choices, levels of participation of player $i$ to the game. Each lattice may be different.

First, let us examine several particular examples.

- $(L, \leq)=\left(2^{N}, \subseteq\right)$. This is the classical notion of game. Each player has two possible actions (participate, not participate), hence $L_{i}=\{0,1\} . L$ is a Boolean lattice.

- $(L, \leq)=\left(3^{N}, \leq\right)$. This case comprises ternary voting games and bi-cooperative games (each $L_{i}$ can be coded as $L_{i}=\{-1,0,1\}$, where 0 means "no participation", -1 means voting or playing against, and 1 means voting or playing in favor), as well as multichoice games with $m=2$, letting $L_{i}=\{0,1,2\}$, with 0 indicating no participation, and 1,2 participation (low and high). In fact, Grabisch [10] distinguishes these two cases, the first one being called bipolar game since the $L_{i}$ 's have a symmetric structure around 0 .

- $(L, \leq)=\left(m^{N}, \leq\right)$, with $L_{i}=\{0,1, \ldots, m\}$. This corresponds to multichoice games as introduced by Hsiao and Raghavan. In this paper we will call them $m$-choice games, and call multichoice game the case where each $L_{i}$ is a linear lattice $L_{i}:=$ $\left\{0,1, \ldots, l_{i}\right\}$ (i.e., the number of levels may be different for each player).

- $(L, \leq)=\left([0,1]^{n}, \leq\right)$. This corresponds to fuzzy games.

Note that the case of global games cannot be recovered by our definition, since the lattice of partitions is not a product lattice.

Let us turn to the interpretation of our definition. We assume that each player $i \in N$ has at his/her disposal a set of elementary or pure actions $j_{1}, \ldots, j_{n_{i}}$. These elementary actions are partially ordered (e.g. in the sense of benefit caused by the action), forming a partially ordered set $\left(\mathcal{J}_{i}, \leq\right)$. Then by virtue of Birkhoff's theorem (see Sec. 2), the set $\left(\mathcal{O}\left(\mathcal{J}_{i}\right), \subseteq\right)$ of downsets of $\mathcal{J}_{i}$ is a distributive lattice denoted $L_{i}$, whose join-irreducible elements correspond to the elementary actions. The bottom action $\perp$ of $L_{i}$ is the action which amounts to do nothing. Hence, each action in $L_{i}$ is either a pure action $j_{k}$ or a combined action $j_{k} \vee j_{k^{\prime}} \vee j_{k^{\prime \prime}} \vee \cdots$ consisting of doing all actions $j_{k}, j_{k^{\prime}}, \ldots$ for player $i$.

For example, assume that players are gardeners who take care of some garden or park. Elementary actions are watering $(\mathrm{W})$, light weeding $(\mathrm{LW})$, careful weeding $(\mathrm{CW})$, and pruning $(\mathrm{P})$. All these actions are benefic for the garden and clearly $\mathrm{LW}<\mathrm{CW}$, but otherwise actions seem to be incomparable. They form the following partially ordered set:

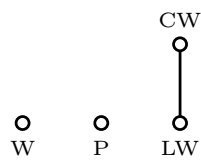

which in turn form the following lattice of possible actions: 


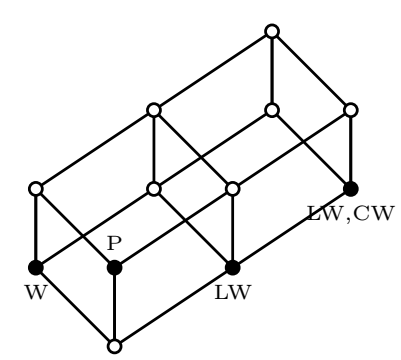

Let us give another interpretation of our framework, borrowed from Faigle and Kern [6]. Let $P:=(N, \leq)$ be a partially ordered set of players, where $\leq$ is a relation of precedence: $i \leq j$ if the presence of $j$ enforces the presence of $i$ in any coalition $S \subseteq N$. Hence, a (valid) coalition of $P$ is a subset $S$ of $N$ such that $i \in S$ and $j \leq i$ entails $j \in S$. Hence, the collection $\mathcal{C}(P)$ of all coalitions of $P$ is the collection of all downsets (ideals) of $P$. A game on $P$ is any function $v: \mathcal{C}(P) \rightarrow \mathbb{R}$ such that $v(\emptyset)=0$.

From this definition, it is possible to recover our structure. For each player $i$ in $N$, let $\mathcal{J}_{i}:=\left\{j_{1}, \ldots, j_{n_{i}}\right\}$ be the set of elementary actions of player $i$. Consider the set of virtual players

$$
N^{\prime}:=\bigcup_{i \in N} \mathcal{J}_{i}
$$

equipped with the partial order $\leq$ induced by the partial orders on each $\mathcal{J}_{i}$. Then coalitions of $\left(N^{\prime}, \leq\right)$ correspond bijectively to elements of $\prod_{i \in N} \mathcal{O}\left(\mathcal{J}_{i}\right)$

\section{Previous works on the Shapley value}

We present in this section the Shapley value defined by Faigle and Kern, and the one defined by Hsiao and Raghavan, together with their axiomatization. A good comparison of these two values can be found in [3]. We present them with our notations, which are rather far from the original ones.

The value introduced by Faigle and Kern is the average of the marginal vectors along all maximal chains in $L$. A maximal chain in a (finite) lattice $L$ is a sequence of elements $C=\{\perp, x, y, z, \ldots, \top\}$ such that $\perp \prec x \prec y \prec z \cdots \prec \top$. We denote by $\mathcal{C}(L)$ the set of all maximal chains on $L$. Then the Shapley value of Faigle and Kern is defined by:

$$
\phi_{\mathrm{FK}}^{v}\left(j_{i}\right):=\frac{1}{|\mathcal{C}(L)|} \sum_{C \in \mathcal{C}(L)}\left[v\left(x_{j_{i}}\right)-v\left(\underline{x_{j_{i}}}\right)\right],
$$

for any join-irreducible element $j_{i}$ of $L_{i}$, and for any $i \in N$. The element $x_{j_{i}}$ is the first in the sequence $C$ containing $j_{i}$ in $\eta\left(x_{j_{i}}\right)$, and $\underline{x}_{j_{i}}$ is its predecessor in the chain $C$. In the vocabulary of Faigle and Kern, maximal chains correspond to what they call feasible ranking of join-irreducible elements (players).

The axiomatic of Faigle and Kern is essentially based on linearity (L) and the unique decomposition of a game on the basis of unanimity games. In this case, a unanimity game $u_{x}$ is defined by, for any $x \in L$ :

$$
u_{x}(y):= \begin{cases}1, & \text { if } y \geq x \\ 0, & \text { otherwise }\end{cases}
$$


Then the coordinates of any game $v$ in this basis are given by the Möbius transform (or dividend) of $v$ [17]. It remains then to fix the Shapley value of any unanimity game by some suitable axioms. They are indicated below.

An element $c \in L$ is a carrier if $v(x \wedge c)=v(x)$, for all $x \in L$.

Carrier axiom (C): If $c$ is a carrier for $V$, then $\sum_{j_{i} \leq c} \phi_{\mathrm{FK}}^{v}\left(j_{i}\right)=v(c)$.

The hierarchical strength of a join-irreducible element $j_{i} \in L_{i}$ with respect to some $x \in L$ is defined by the relative number of maximal chains in $L$ where $x$ is the first occurence of $j_{i}$ in the chain, that is:

$$
h_{x}\left(j_{i}\right):=\frac{1}{|\mathcal{C}(L)|}\left|\left\{C \in \mathcal{C}(L) \mid x_{j_{i}}=x\right\}\right|
$$

Hierarchical strength axiom (HS): For any $x \in L$ and any join-irreducible elements $j_{i}, j_{i^{\prime}}^{\prime} \in \eta(x)$,

$$
h_{x}\left(j_{i}\right) \phi_{\mathrm{FK}}^{u_{x}}\left(j_{i^{\prime}}^{\prime}\right)=h_{x}\left(j_{i^{\prime}}^{\prime}\right) \phi_{\mathrm{FK}}^{u_{x}}\left(j_{i}\right)
$$

Then, under axioms (L), (C) and (HS), the value of the unanimity game $u_{x}$ is uniquely determined:

$$
\phi_{\mathrm{FK}}^{u_{x}}\left(j_{i}\right)= \begin{cases}0, & \text { if } j_{i} \notin \eta(x) \\ h_{x}\left(j_{i}\right) / \sum_{k \in \eta(x)} h_{x}(k), & \text { otherwise. }\end{cases}
$$

We turn to the value proposed by Hsiao and Raghavan, which is limited to $m$-choice games in our terminology. Its construction is similar to the one of Faigle and Kern because it is based also on unanimity games. The main difference is that Hsiao and Raghavan introduced weights for all possible actions of the players, leading to a kind a weighted Shapley value. Let us denote by $w_{1}, \ldots, w_{m}$ the weights of actions $1, \ldots, m$; they are such that $w_{1}<\cdots<w_{m}$. The first axiom is additivity (A) of the value, i.e. $\phi_{\mathrm{HR}}^{v+w}=\phi_{\mathrm{HR}}^{v}+\phi_{\mathrm{HR}}^{w}$. The second axiom is the carrier axiom (C), as for Faigle and Kern. The remaining ones are as follows.

Minimal effort axiom (ME): if $v$ is such that $v(x)=0$ for all $x \nsupseteq y$, then for all players $i$, all action $k_{i}<y_{i}$, we have $\phi_{\mathrm{HR}}^{v}\left(k_{i}\right)=0$.

Weight axiom (W): If $v:=\alpha u_{x}$ for some $\alpha>0$, then $\phi_{\mathrm{HR}}^{v}\left(x_{i}\right)$ is proportional to $w_{x_{i}}$, for all $i \in N$.

Using these axioms, it can be shown that, $j_{i}$ denoting action $j$ for player $i$ :

$$
\phi_{\mathrm{HR}}^{u_{x}}\left(j_{i}\right)= \begin{cases}\frac{w_{j}}{\sum_{i \in N} w_{x_{i}}}, & \text { if } j=x_{i} \\ 0, & \text { otherwise }\end{cases}
$$

Let us discuss these values. As remarked by Faigle and Kern, since the problem of computing the number of maximal chains in a partially ordered set is a $\sharp \mathrm{P}$-complete counting problem, it is doubtful whether an efficient algorithm could exist to compute 
exactly $\phi_{\mathrm{FK}}$. For multichoice games, the number of maximal chains is, with our notation $[3,6]$ :

$$
|\mathcal{C}(L)|=\frac{\left(\sum_{i \in N} l_{i}\right) !}{\prod_{i \in N}\left(l_{i} !\right)}=\left(\begin{array}{c}
l \\
l_{1}
\end{array}\right)\left(\begin{array}{c}
l-l_{1} \\
l_{2}
\end{array}\right)\left(\begin{array}{c}
l-l_{1}-l_{2} \\
l_{3}
\end{array}\right) \cdots 1,
$$

with $l:=\prod_{i \in N} l_{i}$. For 5 players having each 3 actions (3-choice game), this gives already $(15) ! / 6^{5}=168,168,000$. The same remark applies to $\phi_{\mathrm{HR}}$, since its explicit expression given in [14] is very complicated. In [3], Branzei et al. have shown that $\phi_{\mathrm{FK}}$ and $\phi_{\mathrm{HR}}$ do not coincide in general. Even more, one can find examples where for no system of weights the two values can coincide.

Concerning the axiomatic, the one of Faigle and Kern is very simple, although the meaning of the (HS) axiom is not completely clear, at least in our framework of games on lattices (recall that this axiomatic was primarily intended for games with precedence constraints). The axiomatic of Hsiao and Raghavan is simple and clear, but they need weights on action, which are necessarily all different, so one could ask about what if no weight is wanted, and what do precisely mean these weights (in particular, what is the exact difference between $w_{j}$ and $v\left(j_{i}\right)$ ?).

In the next section, we present an alternative view.

\section{Axiomatic of the Shapley value for multichoice games}

Our approach will take a different way. We do not use unanimity games, but introduce axioms similar to the original ones of Shapley, adding them one by one as Weber in [19], to see the exact effect of each axiom. Surprisingly, we will come up with a value which is very near the classical Shapley value, and very simple to compute.

\subsection{Notations, differential and cumulative values}

We recall that for every player $i, L_{i}$ is a linear lattice denoted $L_{i}:=\left\{0,1,2, \ldots, l_{i}\right\}$. The set $\mathcal{J}(L)$ of join-irreducible elements (or virtual players in the framework of Faigle and Kern) of $L$ is $\left\{\left(0_{1}, \ldots, 0_{i-1}, k_{i}, 0_{i+1}, \ldots, 0_{n}\right) \mid i \in N, k \in L_{i} \backslash\{0\}\right\}$; hence each joinirreducible element corresponds to a single player playing at a given level. Since we use them constantly in the following, we will often adopt the shorthand $\widetilde{k}_{i}$ for $\left(0_{1}, \ldots, 0_{i-1}, k_{i}, 0_{i+1}, \ldots, 0_{n}\right)$.

Our aim is to define the Shapley value for each join-irreducible element $\widetilde{k}_{i}$. A first approach would be to define the Shapley value for $\widetilde{k}_{i}$ as a kind of average contribution of player $i$ playing at level $k$, compared to the situation where $i$ plays at level $k-1$. We call this a differential value, which we denote by $\phi\left(k_{i}\right)$. A differential value obviously satisfies what could be called a differential null axiom, saying that $\phi\left(k_{i}\right)=0$ whenever player $i$ is such that $v\left(x_{-i}, k_{i}\right)=v\left(x_{-i},(k-1)_{i}\right)$ for all $x_{-i} \in L_{-i}$, using our notation for compound vectors (see Section 2).

A careful look at the previous axiomatizations of Faigle and Kern, and Hsiao and Raghavan, show that their value are differential. This is due to the carrier axiom, which could be implied by the differential null axiom and a suitable efficiency axiom (see also formula (1), which obviously satisfies the differential null axiom). 
However, if we stick to the idea that the Shapley value for $k_{i}$ should be a reward for player $i$ having played at level $k$, it should express an average of the contribution of player $i$ playing at level $k$, but compared to the situation where $k$ does not participate. Roughly speaking, this amounts to sum all differential values from the first level to the $k$ th level. Hence, such a value could be called a cumulative value, and to our opinion, it is the only one of interest, the differential value being merely an intermediate step of computation. We denote by $\Phi\left(k_{i}\right)$ the cumulative value for player $i$ playing at level $k$.

Our position is to give directly an axiomatization of the cumulative Shapley value, which in the sequel will be called simply "Shapley value". It is possible however to derive a similar axiomatization for the differential value (see [10] for the case of $m$-choice games).

\subsection{The axiomatic of the (cumulative) Shapley value}

Let us give first the following definitions generalizing the ones given for classical games.

- for some $k \in L_{i}, k \neq 0$, player $i$ is said to be $k$-null (or simply $k_{i}$ is null) for $v \in \mathcal{G}(L)$ if $v\left(x, k_{i}\right)=v\left(x, 0_{i}\right), \forall x \in L_{-i}$.

- for some $k \in L_{i}, k \neq 0$, player $i$ is said to be $k$-dummy (or simply $k_{i}$ is dummy) for $v \in \mathcal{G}(L)$ if $v\left(x, k_{i}\right)=v\left(x, 0_{i}\right)+v\left(\widetilde{k_{i}}\right), \forall x \in L_{-i}$.

- $v \in \mathcal{G}(L)$ is said to be monotone if $v(x) \leq v(y)$, for all $x, y$ in $L$ such that $x \leq y$.

This enables to introduce the following axioms:

Null axiom $(\mathbf{N}): \forall v \in \mathcal{G}(L)$, for all null $k_{i}, \Phi^{v}\left(k_{i}\right)=0$.

$\operatorname{Dummy} \operatorname{axiom}(\mathbf{D}): \forall v \in \mathcal{G}(L)$, for all dummy $k_{i}, \Phi^{v}\left(k_{i}\right)=v\left(\widetilde{k}_{i}\right)$.

As for classical games, the dummy axiom implies the null axiom. Indeed, assume $k_{i}$ is null. Then $v\left(\widetilde{k_{i}}\right)=v(0)=0$, so that $v\left(x, k_{i}\right)=v\left(x, 0_{i}\right)+v\left(\widetilde{k_{i}}\right)$ holds, i.e. $k_{i}$ is dummy. Then $\Phi_{v}\left(k_{i}\right)=v\left(\widetilde{k}_{i}\right)=0$, which proves that $(\mathrm{N})$ holds.

Monotonicity axiom (M): $\forall v \in \mathcal{G}(L)$, if $v$ is monotone, then $\Phi^{v}\left(k_{i}\right) \geq 0$, for all $\widetilde{k}_{i} \in \mathcal{J}(L)$.

Linearity axiom (L): For all $\widetilde{k_{i}} \in \mathcal{J}(L), \Phi\left(k_{i}\right)$ is linear on the set of games $\mathcal{G}(L)$, which directly implies

$$
\Phi^{v}\left(k_{i}\right)=\sum_{x \in L} a_{x}^{k_{i}} v(x), \quad \text { with } a_{x}^{k_{i}} \in \mathbb{R}
$$

Proposition 1 Under axioms $(L)$ and $(N), \forall v \in \mathcal{G}(L)$, for all $\widetilde{k}_{i} \in \mathcal{J}(L)$,

$$
\Phi^{v}\left(k_{i}\right)=\sum_{x \in L_{-i}} p_{x}^{k_{i}}\left[v\left(x, k_{i}\right)-v\left(x, 0_{i}\right)\right], \quad \text { with } p_{x}^{k_{i}} \in \mathbb{R} .
$$


Proof: It is clear that the above formula satisfies the axioms. Conversely, assuming $k_{i}$ is null,

$$
\begin{aligned}
\Phi_{v}\left(k_{i}\right) & =\sum_{x \in L} a_{x}^{k_{i}} v(x) \\
& =\sum_{x \in L_{-i}}\left[a_{\left(x, 0_{i}\right)}^{k_{i}} v\left(x, 0_{i}\right)+\cdots+a_{\left(x, l_{i}\right)}^{k_{i}} v\left(x, l_{i}\right)\right] \\
& =\sum_{x \in L_{-i}} v\left(x, 0_{i}\right)\left[a_{\left(x, 0_{i}\right)}^{k_{i}}+a_{\left(x, k_{i}\right)}^{k_{i}}\right]+\sum_{x \in L_{-i}} \sum_{j \neq 0, k} a_{\left(x, j_{i}\right)}^{k_{i}} v\left(x, j_{i}\right) .
\end{aligned}
$$

Consider $v^{\prime} \in \mathcal{G}\left(L_{-i}\right)$ and extend it to $\mathcal{G}(L)$ :

$$
v\left(x, j_{i}\right)= \begin{cases}v^{\prime}(x), & \text { if } j=k, 0 \\ 0, & \text { otherwise }\end{cases}
$$

Then $k_{i}$ is null for $v$, hence (4) applies and reduces to:

$$
\Phi_{v}\left(k_{i}\right)=\sum_{x \in L_{-i}} v^{\prime}(x)\left[a_{\left(x, 0_{i}\right)}^{k_{i}}+a_{\left(x, k_{i}\right)}^{k_{i}}\right]=0 .
$$

This implies $a_{\left(x, k_{i}\right)}^{k_{i}}=-a_{\left(x, 0_{i}\right)}^{k_{i}}$. Introducing this in (4) we get:

$$
\Phi_{v}\left(k_{i}\right)=0=\sum_{x \in L_{-i}} \sum_{j \neq 0, k} a_{\left(x, j_{i}\right)}^{k_{i}} v\left(x, j_{i}\right) .
$$

Since this must hold for any game, we deduce that $a_{\left(x, j_{i}\right)}^{k_{i}}=0, \forall j \neq 0, k$. Letting $p_{x}^{k_{i}}:=a_{\left(x, k_{i}\right)}^{k_{i}}$, the result is proven.

Proposition 2 Under axioms $(L)$ and $(D), \forall v \in \mathcal{G}(L)$, for all $\widetilde{k_{i}} \in \mathcal{J}(L)$,

$$
\Phi^{v}\left(k_{i}\right)=\sum_{x \in L_{-i}} p_{x}^{k_{i}}\left[v\left(x, k_{i}\right)-v\left(x, 0_{i}\right)\right], \quad \text { with } p_{x}^{k_{i}} \in \mathbb{R}, \text { and } \sum_{x \in L_{-i}} p_{x}^{k_{i}}=1 .
$$

Proof: We consider the unanimity game $u_{k_{i}}$ defined by

$$
u_{k_{i}}(x)= \begin{cases}1, & \text { if } x \geq k_{i} \\ 0, & \text { otherwise }\end{cases}
$$

$k_{i}$ is dummy since $u_{k_{i}}\left(x, k_{i}\right)=1=u_{k_{i}}\left(x, 0_{i}\right)+u_{k_{i}}\left(\widetilde{k_{i}}\right)$. Hence

$$
\Phi_{u_{k_{i}}}\left(k_{i}\right)=u_{k_{i}}\left(\widetilde{k_{i}}\right)=1=\sum_{x \in L_{-i}} p_{x}^{k_{i}} \cdot 1
$$

which proves the result. 
Proposition 3 Under axioms $(L),(N)$ and $(M), \forall v \in \mathcal{G}(L)$, for all $\widetilde{k_{i}} \in \mathcal{J}(L)$,

$$
\Phi^{v}\left(k_{i}\right)=\sum_{x \in L_{-i}} p_{x}^{k_{i}}\left[v\left(x, k_{i}\right)-v\left(x, 0_{i}\right)\right], \quad \text { with } p_{x}^{k_{i}} \geq 0 .
$$

Proof: Let choose some $y \in L$ and define by analogy with classical games

$$
\hat{u}_{y}(x)= \begin{cases}1, & \text { if } x \geq y, x \neq y \\ 0, & \text { else. }\end{cases}
$$

By definition, $\hat{u}_{y}$ is monotone. Letting $y=\left(x_{0}, 0_{i}\right)$ for some $x_{0} \in L_{-i}$, and applying Prop. 1, we get:

$$
\begin{aligned}
\Phi_{\hat{u}_{\left(x_{0}, 0_{i}\right.}}\left(k_{i}\right) & =\sum_{x \in L_{-i}} p_{x}^{k_{i}}\left[\hat{u}_{\left(x_{0}, 0_{i}\right.}\left(x, k_{i}\right)-\hat{u}_{\left(x_{0}, 0_{i}\right.}\left(x, 0_{i}\right)\right] \\
& =p_{x_{0}}^{k_{i}} \geq 0 .
\end{aligned}
$$

As a consequence, one can deduce from Propositions 2 and 3 that under axioms (L),(D) and $(\mathrm{M})$, for every join-irreducible $\widetilde{k}_{i},\left(p_{x}^{k_{i}}\right)_{x \in L_{-i}}$ will be a probability distribution.

The next axiom enables an easier computation of coefficients $p_{x}^{k_{i}}$ while reducing their number:

Invariance axiom (I): Let us consider two games $v_{1}, v_{2}$ of $\mathcal{G}(L)$ such that for some $i$ in $N$,

$$
\begin{aligned}
& v_{1}\left(x, k_{i}\right)=v_{2}\left(x,(k-1)_{i}\right), \forall x \in L_{-i}, \forall k \in L_{i}, k>1 \\
& v_{1}\left(x, 0_{i}\right)=v_{2}\left(x, 0_{i}\right), \forall x \in L_{-i} .
\end{aligned}
$$

Then $\Phi^{v_{1}}\left(k_{i}\right)=\Phi^{v_{2}}\left((k-1)_{i}\right), 1<k \leq l_{i}$.

The axiom says that when a game $v_{2}$ is merely a shift of another game $v_{1}$ concerning player $i$, the Shapley values are the same for this player. This implies that the way of computing $v$ does not depend on the level $k$, as shown in the next proposition.

Proposition 4 Under axioms $(L),(N)$ and $(I), \forall v \in \mathcal{G}(L)$, for all join-irreducible $k_{i}$,

$$
\Phi^{v}\left(k_{i}\right)=\sum_{x \in L_{-i}} p_{x}^{i}\left[v\left(x, k_{i}\right)-v\left(x, 0_{i}\right)\right], \quad \text { with } p_{x}^{i} \in \mathbb{R} .
$$

Proof: We have for $k>1$

$$
\begin{aligned}
\Phi_{v_{1}}\left(k_{i}\right) & =\sum_{x \in L_{-i}} p_{x}^{k_{i}}\left[v_{1}\left(x, k_{i}\right)-v_{1}\left(x, 0_{i}\right)\right] \\
& =\sum_{x \in L_{-i}} p_{x}^{k_{i}}\left[v_{2}\left(x,(k-1)_{i}\right)-v_{2}\left(x, 0_{i}\right)\right] \\
\Phi_{v_{2}}\left((k-1)_{i}\right) & =\sum_{x \in L_{-i}} p_{x}^{(k-1)_{i}}\left[v_{2}\left(x,(k-1)_{i}\right)-v_{2}\left(x, 0_{i}\right)\right],
\end{aligned}
$$


which proves the result.

Let us now introduce a symmetry axiom, which is an adaptation of the classical symmetry axiom. The difficulty here is that since the $L_{i}$ 's could be different, applying directly the classical symmetry axiom may lead to meaningless expressions. In this purpose, we introduce a subspace of $\mathcal{G}(L)$ :

$$
\mathcal{G}_{0}(L):=\{v \in \mathcal{G}(L) \mid v(x)=0, \forall x \notin \Gamma(L)\},
$$

where we recall that $\Gamma(L)=\left\{0_{1}, l_{1}\right\} \times\left\{0_{2}, l_{2}\right\} \times \cdots \times\left\{0_{n}, l_{n}\right\}$ is the set of vertices of $L$. For any $x$ in $\Gamma(L)$ and any permutation $\sigma$ on $N$, we define $x^{\sigma}:=\left(x_{1}^{\sigma}, \ldots, x_{n}^{\sigma}\right)$ by

$$
x_{i}^{\sigma}:= \begin{cases}0_{i}, & \text { if } x_{\sigma(i)}=0_{\sigma(i)} \\ l_{i}, & \text { if } x_{\sigma(i)}=l_{\sigma(i)}\end{cases}
$$

Besides, for any $v \in \mathcal{G}_{0}(L)$, we denote by $v^{\sigma}$ the game in $\mathcal{G}_{0}(L)$ such that $v^{\sigma}(x):=v\left(x^{\sigma}\right)$, for any $x$ in $\Gamma(L)$. When all $l_{i}$ 's are different, observe that $x^{\sigma}$ is a vertice of $\Gamma(L)$, contrary to $\sigma(x):=\left(x_{\sigma(1)}, \ldots, x_{\sigma(n)}\right)$, as well as $v^{\sigma}$ is a game in $\mathcal{G}_{0}(L)$ while $v \circ \sigma$ is not. Let us take for example $L:=\{0,1,2\} \times\{0,1,2,3,4\} \times\{0,1,2,3\}$, and

\begin{tabular}{|c|lll|}
\hline$i$ & 1 & 2 & 3 \\
$\sigma(i)$ & 2 & 3 & 1 \\
\hline
\end{tabular}

Then $(2,0,0)^{\sigma}=(0,0,3),(2,0,3)^{\sigma}=(0,4,3)$.

Symmetry axiom (S): Let $\sigma$ be a permutation on $N$. Then for any game $v$ in $\mathcal{G}_{0}(L)$, and any $i$ in $N$,

$$
\Phi^{v^{\sigma^{-1}}}\left(l_{i}^{\sigma}\right)=\Phi^{v}\left(l_{i}\right)
$$

Thus, as for classical games, this axiom says that the computation of Shapley value should not depend on the labelling of the players. Finally, we give the last axiom:

Efficiency axiom (E): $\forall v \in \mathcal{G}(L), \sum_{i \in N} \Phi^{v}\left(l_{i}\right)=v\left(l_{1}, \ldots, l_{n}\right)$.

Theorem 1 Under axioms $(L),(D),(M),(I),(S)$ and $(E), \forall v \in \mathcal{G}(L)$, for all $\widetilde{k_{i}} \in \mathcal{J}(L)$,

$$
\Phi^{v}\left(k_{i}\right)=\sum_{x \in \Gamma\left(L_{-i}\right)} \frac{(n-h(x)-1) ! h(x) !}{n !}\left[v\left(x, k_{i}\right)-v\left(x, 0_{i}\right)\right],
$$

where $h(x):=\left|\left\{j \in N \backslash i \mid x_{j}=l_{j}\right\}\right|$.

Proof: Let $v$ be a game in $\mathcal{G}_{0}(L)$ and let $\sigma$ be a transposition of $N$, that is to say a permutation which only exchanges two players $i$ and $j$. This implies $\sigma=\sigma^{-1}$. Then by (S) we have $\Phi^{v}\left(l_{i}\right)=\Phi^{v^{\sigma}}\left(l_{j}\right)$, which writes, using axioms (L), (D), (M) and (I), and Prop. 3 and 4 :

$$
\sum_{x \in \Gamma\left(L_{-i}\right)} p_{x}^{i}\left[v\left(x, l_{i}\right)-v\left(x, 0_{i}\right)\right]=\sum_{x \in \Gamma\left(L_{-j}\right)} p_{x}^{j}\left[v\left(\left(x, l_{j}\right)^{\sigma}\right)-v\left(\left(x, 0_{j}\right)^{\sigma}\right)\right]
$$


which can be rewritten as

$$
\begin{aligned}
\sum_{x \in \Gamma\left(L_{-i, j}\right)} \sum_{x_{j} \in\left\{0, l_{j}\right\}} p_{x, x_{j}}^{i}\left[v\left(x, l_{i}, x_{j}\right)-v\left(x, 0_{i}, x_{j}\right)\right]= \\
\quad \sum_{x \in \Gamma\left(L_{-i, j}\right)} \sum_{x_{i} \in\left\{0, l_{i}\right\}} p_{x, x_{i}}^{j}\left[v\left(\left(x, x_{i}, l_{j}\right)^{\sigma}\right)-v\left(\left(x, x_{i}, 0_{j}\right)^{\sigma}\right)\right] .
\end{aligned}
$$

If $x \in \Gamma\left(L_{-i, j}\right)$, then $\left(x, x_{i}, l_{j}\right)^{\sigma}=\left(x, l_{i}, x_{j}^{\prime}\right)$, and $\left(x, x_{i}, 0_{j}\right)^{\sigma}=\left(x, 0_{i}, x_{j}^{\prime}\right)$, where $x_{j}^{\prime}$ is of the same nature than $x_{i}$, (i.e. $x_{j}^{\prime}=0$ iff $x_{i}=0$, and $x_{j}^{\prime}=l_{j}$ iff $x_{i}=l_{i}$ ). Consequently, as the above equalities are true for any $v \in \mathcal{G}_{0}(L)$, we can identify the term of the first member coefficient of which is $p_{x, x_{j}}^{i}$, with the term of the second member coefficient of which is $p_{x, x_{i}}^{j}$, such that $x_{i}$ and $x_{j}$ are of the same nature. This gives equality between these coefficients.

By taking into account all transpositions of $N$, for any $x$ in $\Gamma\left(L_{-i, j, l}\right)$, we write

$$
\begin{aligned}
p_{x, x_{j}, x_{l}}^{i} & =p_{x, x_{i}^{\prime}, x_{l}}^{j} \text { where } x_{i}^{\prime} \text { of the same nature than } x_{j}, \\
& =p_{x, x_{i}^{\prime}, x_{j}^{\prime}}^{l} \text { where } x_{j}^{\prime} \text { of the same nature than } x_{l} ; \\
\text { besides, } p_{x, x_{j}, x_{l}}^{i} & =p_{x, x_{i}^{\prime \prime}, x_{j}}^{l} \text { where } x_{i}^{\prime \prime} \text { of the same nature than } x_{l} \text { and thus of } x_{j}^{\prime} .
\end{aligned}
$$

As a result, for all $l \neq i, j$ and for all $x \in \Gamma\left(L_{-i, j, l}\right), p_{x, x_{i}^{\prime}, x_{j}^{\prime}}^{l}=p_{x, x_{i}, x_{j}}^{l}$ whenever $x_{i}$ and $x_{j}^{\prime}$ have the same nature, as well as $x_{i}^{\prime}$ and $x_{j}$. Consequently, for the computation of $p_{x}^{l}, x \in$ $L_{-l}$, any permutation being a composition of transpositions, indices of components $0_{i}$ and $l_{i}$ of $x$ have no importance as long as the cardinality $h(x)=\left|\left\{i \in N \backslash l \mid x_{i}=l_{i}\right\}\right|$ is the same. Therefore, we will use a new notation for $p_{x}^{l}$ :

$$
p_{m}^{l}:=p_{x}^{l} \text {, where } m=h(x) .
$$

Moreover, it is clear that for all $i, j \in N$, for all $m \in\{0, \ldots, n-1\}, p_{m}^{i}=p_{m}^{j}$, due to the effect of the transposition $i \leftrightarrow j$. It follows that one can write $p_{m}$ instead of $p_{m}^{i}, i \in N$.

Now, by efficiency axiom, we have $\sum_{i \in N} \sum_{x \in L_{-i}} p_{x}^{i}\left[v\left(x, l_{i}\right)-v\left(x, 0_{i}\right)\right]=v\left(l_{1}, \ldots, l_{n}\right)$. Assuming $v$ is a game in $\mathcal{G}_{0}(L)$, this gives the following equation:

$$
\sum_{i \in N} \sum_{m=0}^{n-1} \sum_{\substack{x \in L_{-i}, h(x)=m}} p_{m}\left[v\left(x, l_{i}\right)-v\left(x, 0_{i}\right)\right]=v\left(l_{1}, \ldots, l_{n}\right) .
$$

Le us denote $\mathcal{G}\left(2^{N}\right)$ the set of classical games on $N$ and $v \mapsto \tilde{v}$ the canonical isomorphism from $\mathcal{G}_{0}(L)$ to $\mathcal{G}\left(2^{N}\right)$, i.e. for all $S \in 2^{N}$

$$
\tilde{v}(S):=v(s), \quad \text { with } s_{i}=\left\{\begin{array}{ll}
l_{i}, & \text { if } i \in S \\
0_{i}, & \text { else }
\end{array}, \forall i \in N\right.
$$

Observe that, through this mapping, Eq. (5) becomes

$$
\sum_{i \in N} \sum_{m=0}^{n-1} \sum_{\substack{S \subseteq N \backslash i,|S|=m}} p_{m}[\tilde{v}(S \cup i)-\tilde{v}(S)]=\tilde{v}(N) .
$$


We recognize here the classical efficiency axiom, from which we deduce that coefficients $p_{m}$ 's are nothing else that the well-known Shapley coefficients $p_{m}=\alpha_{m}^{1}(n):=\frac{(n-m-1) ! m !}{n !}$ for all $m \in\{0 \ldots, n-1\}$.

As a consequence, through inverse of the above isomorphism, we easily obtain the expression of the previous $p_{x}^{i}$ when $x \in \Gamma\left(L_{-i}\right)$ :

$$
p_{x}^{i}=\frac{(n-h(x)-1) ! h(x) !}{n !} .
$$

Finally, as $\left(p_{x}^{i}\right)_{x \in L_{-i}}$ is a probability distribution, and since we know that $\sum_{S \subseteq N \backslash i} \alpha_{|S|}^{1}=$ $\sum_{x \in \Gamma\left(L_{-i}\right)} p_{x}^{i}=1$, it follows that $p_{x}^{i}=0$ for all $x \in L_{-i} \backslash \Gamma\left(L_{-i}\right)$.

REMARK 1: It is possible to give a rather different formulation suggested by the proof of Th. 1 by introducing the following axioms:

Symmetry axiom for classical games (CS): Let $\sigma$ be a permutation on $N$. Then for any game $\nu$ in $\mathcal{G}\left(2^{N}\right)$, and any $i$ in $N, \Phi^{\nu \circ \sigma^{-1}}(\sigma(i))=\Phi^{\nu}(i)$.

Full participation axiom (FP): For any game $v$ in $\mathcal{G}_{0}(L)$, and any $i$ in $N$, $\Phi^{v}\left(l_{i}\right)=\Phi^{\tilde{v}}(i)$.

Consequently, axiom (S) being equivalent to the pair ((CS), (FP)) under axioms $(\mathrm{L}),(\mathrm{D}),(\mathrm{M}),(\mathrm{I}),(\mathrm{E})$, the required theorem can also be proven with these axioms and (CS), (FP) instead of $(\mathrm{S})$.

\section{Towards the general case}

In this section, we present first ideas to define a Shapley value for the general case, where the $L_{i}$ 's are finite distributive, as a basis for future research. Our aim is to obtain $\Phi^{v}\left(x_{i}\right)$, for any $x_{i} \in L_{i}, x_{i} \neq \perp_{i}$, which should represent the contribution of doing action $x_{i}$ instead of nothing for player $i$. We denote as usual the top and bottom elements of each lattice $L_{i}$ by $\top_{i}, \perp_{i}$.

A first approach is to adapt the previous axiomatization for multichoice games to the general case. This can be done under the restriction that in each $L_{i}$, the bottom element $\perp_{i}$ has a unique successor, denoted by $1_{i}$ (in other words, $1_{i}$ is the unique atom of $L_{i}$ ). Also, for any $x_{i} \in L_{i}, x_{i} \neq \perp_{i}, \underline{x}_{i}:=\bigwedge\left\{y_{i} \in L_{i} \mid y_{i} \prec x_{i}\right\}$, i.e., $\underline{x}_{i}$ is the infimum of all predecessors of $x_{i}$. The following axioms and definitions are direct generalizations of the previous ones:

- For some $x_{i} \in L_{i} \backslash \perp_{i}$, player $i$ is $x_{i}$-null (or simply $x_{i}$ is null) for $v \in \mathcal{G}(L)$ if $v\left(x, x_{i}\right)=v\left(x, \perp_{i}\right), \forall x \in L_{-i}$.

- For some $x_{i} \in L_{i} \backslash \perp_{i}$, player $i$ is $x_{i}$-dummy (or simply $x_{i}$ is dummy) for $v \in \mathcal{G}(L)$ if $v\left(x, x_{i}\right)=v\left(x, \perp_{i}\right)+v\left(\perp_{-i}, x_{i}\right), \forall x \in L_{-i}$.

- $\mathbf{N u l l}$ axiom (N): $\forall v \in \mathcal{G}(L)$, for all null $x_{i}, \phi^{v}\left(x_{i}\right)=0$.

- Dummy axiom (D): $\forall v \in \mathcal{G}(L)$, for all dummy $x_{i}, \phi^{v}\left(x_{i}\right)=v\left(\perp_{-i}, x_{i}\right)$. 
- Monotonicity axiom (M): $\forall v \in \mathcal{G}(L)$, if $v$ is monotone, then $\Phi^{v}\left(x_{i}\right) \geq 0$, for every player $i, x_{i} \neq \perp_{i}$.

- Linearity axiom (L): For all $x_{i} \in L_{i}, x_{i} \neq \perp_{i}, \Phi^{v}\left(x_{i}\right)$ is linear on $\mathcal{G}(L)$.

- Invariance axiom (I): Let us consider two games $v_{1}, v_{2} \in \mathcal{G}(L)$ such that for some $i \in N$,

$$
\begin{aligned}
v_{1}\left(y, x_{i}\right) & =v_{2}\left(y, \underline{x}_{i}\right), \forall y \in L_{-i}, \forall x_{i}>1_{i} \\
v_{1}\left(y, \perp_{i}\right) & =v_{2}\left(y, \perp_{i}\right), \forall y \in L_{-i} .
\end{aligned}
$$

Then $\Phi^{v_{1}}\left(x_{i}\right)=\Phi^{v_{2}}\left(\underline{x}_{i}\right), x_{i}>1_{i}$.

- Symmetry axiom (S): Let $\sigma$ be a permutation on $N$. Then for any game $v \in$ $\mathcal{G}_{0}(L)$ and any $i \in N$,

$$
\Phi^{v^{\sigma^{-1}}}\left(\top_{i}^{\sigma}\right)=\Phi^{v}\left(\top_{i}\right)
$$

with same notations as in previous section.

- Efficiency axiom (E): $\forall v \in \mathcal{G}(L), \sum_{i \in N} \Phi^{v}\left(\top_{i}\right)=v(\top)$.

Using the same schemata of proofs as for multichoice games, we come up with the following result:

Theorem 2 Under axioms $(L),(D),(M),(I),(S)$ and $(E)$, for all $v \in \mathcal{G}(L)$, for all $x_{i} \in L_{i}, x_{i} \neq \perp_{i}$,

$$
\Phi^{v}\left(x_{i}\right)=\sum_{y \in \Gamma\left(L_{-i}\right)} \frac{(n-h(y)-1) ! h(y) !}{n !}\left[v\left(y, x_{i}\right)-v\left(y, \perp_{i}\right)\right],
$$

where $h(y):=\left|\left\{j \in N \backslash i \mid x_{j}=\top_{j}\right\}\right|$.

Although the result is appealing by its simplicity, it suffers from the restriction imposed on the $L_{i}$ 's, and by the fact the axiom (I) becomes questionable. Also, the role of join-irreducible elements as a basic element of the construction has disappeared, which is not in accordance with our interpretation of games on lattice, as given in Section 3.

Based on preceding remarks, we suggest an alternative approach, which goes in several steps, and starts from join-irreducible elements.

1. For any join-irreducible element $x_{i} \in L_{i}$, we compute the differential Shapley value $\phi^{v}\left(x_{i}\right)$, expressing the contribution of doing action $x_{i}$ instead of the predecessor of action $x_{i}$ for player $i$. Since the predecessor of $x_{i}$ is unique iff $x_{i}$ is a join-irreducible element, this makes sense.

2. We compute $\phi^{v}\left(x_{i}\right)$ for any $x_{i} \in L_{i}$, considering its unique irredundant decomposition into join-irreducible elements (see Sec. 2). This unique decomposition always exists since $L$ is distributive.

3. We compute $\Phi^{v}\left(x_{i}\right)$ by cumulating the differential Shapley values between $x_{i}$ and $\perp_{i}$. 
To bring this approach to an operational state, first an axiomatization is needed for defining the differential Shapley value for join-irreducible elements. The second problem is how to use the irredundant decomposition of $x_{i}$ to compute $\phi^{v}\left(x_{i}\right)$. We suggest the following:

$$
\phi^{v}\left(x_{i}\right)=\sum_{j_{i} \in \eta\left(x_{i}\right)} \phi^{v}\left(j_{i}\right)+I^{v}\left(\eta\left(x_{i}\right)\right)
$$

where $I^{v}(S)$ is the interaction among elements of $S \subseteq L_{i}$. The interaction represents the effect of joining elements. For example, for two join-irreducible elements $j_{i}, k_{i}$ :

- $I^{v}\left(\left\{j_{i}, k_{i}\right\}\right)=0$ if the worth of $j_{i} \vee k_{i}$ is the sum of the worths of $j_{i}$ and $k_{i}$

- $I^{v}\left(\left\{j_{i}, k_{i}\right\}\right)>0($ resp. $<0)$ if the worth of $j_{i} \vee k_{i}$ is greater (resp. smaller) than the sum of the worths of $j_{i}$ and $k_{i}$.

The first appearance of the notion of interaction for classical games is due to Owen [16] under the name "co-value". It was rediscovered in a different context by Murofushi and Soneda [15], and generalized by Grabisch [9]. An axiomatization of interaction has been done by Grabisch and Roubens [13], and a general definition for games on lattices has been recently given by Grabisch and Labreuche [12].

We leave the complete setting of this approach for future research.

\section{References}

[1] J. M. Bilbao. Cooperative games on combinatorial structures. Kluwer Academic Publishers, 2000.

[2] J. M. Bilbao, J. R. Fernandez, A. Jiménez Losada, and E. Lebrón. Bicooperative games. In J. M. Bilbao, editor, Cooperative games on combinatorial structures. Kluwer Acad. Publ., 2000.

[3] R. Branzei, D. Dimitrov, and S. Tijs. Models in cooperative game theory: crisp, fuzzy and multichoice games. Springer Verlag, 2005.

[4] D. Butnariu and E. P. Klement. Triangular norm-based measures and games with fuzzy coalitions. Kluwer Academic Publishers, 1993.

[5] B. A. Davey and H. A. Priestley. Introduction to Lattices and Orders. Cambridge University Press, 1990.

[6] U. Faigle and W. Kern. The Shapley value for cooperative games under precedence constraints. Int. J. of Game Theory, 21:249-266, 1992.

[7] D. Felsenthal and M. Machover. Ternary voting games. Int. J. of Game Theory, 26:335-351, 1997.

[8] I. Gilboa and E. Lehrer. Global games. Int. J. of Game Theory, 20:129-147, 1991.

[9] M. Grabisch. $k$-order additive discrete fuzzy measures and their representation. Fuzzy Sets and Systems, 92:167-189, 1997. 
[10] M. Grabisch. An axiomatization of the Shapley value and interaction index for games on lattices. In SCIS-ISIS 2004, 2nd Int. Conf. on Soft Computing and Intelligent Systems and 5th Int. Symp. on Advanced Intelligent Systems, Yokohama, Japan, September 2004.

[11] M. Grabisch. The Shapley value for games on lattices (l-fuzzy games). In Int. Conf. on Fuzzy Sets and Soft Computing in Economics and Finance, St. Petersburg, Russia, June 2004.

[12] M. Grabisch and Ch. Labreuche. Derivative of functions over lattices as a basis for the notion of interaction between attributes. Discrete Applied Maths., to appear.

[13] M. Grabisch and M. Roubens. An axiomatic approach to the concept of interaction among players in cooperative games. Int. Journal of Game Theory, 28:547-565, 1999.

[14] C. R. Hsiao and T. E. S. Raghavan. Shapley value for multichoice cooperative games, I. Games and Economic Behavior, 5:240-256, 1993.

[15] T. Murofushi and S. Soneda. Techniques for reading fuzzy measures (III): interaction index. In 9th Fuzzy System Symposium, pages 693-696, Sapporo, Japan, May 1993. In Japanese.

[16] G. Owen. Multilinear extensions of games. Management Sci., 18:64-79, 1972.

[17] G. C. Rota. On the foundations of combinatorial theory I. Theory of Möbius functions. Zeitschrift für Wahrscheinlichkeitstheorie und Verwandte Gebiete, 2:340-368, 1964. Also in: J.P.S. Kung, editor, Gian-Carlo Rota in Combinatorics: Introductory papers and commentaries, pages 3-31. Birkhäuser, Boston, 1995.

[18] S. Tijs, R. Branzei, S. Ishihara, and S. Muto. On cores and stable sets for fuzzy games. Fuzzy Sets and Systems, 146:285-296, 2004.

[19] R. J. Weber. Probabilistic values for games. In A. E. Roth, editor, The Shapley Value. Essays in Honor of Lloyd S. Shapley, pages 101-119. Cambridge University Press, 1988. 